Research Paper

\title{
Presentation of a method at the Exploration Stage according to IDEAL: Percutaneous nephrolithotomy (PCNL) under local infiltrative anesthesia is a feasible and effective method - retrospective analysis of 439 patients
}

Thorsten H. Ecke ${ }^{1}$, Dimitri Barski², Guido Weingart ${ }^{3}$, Carsten Lange ${ }^{1}$, Steffen Hallmann ${ }^{1}$, Jürgen Ruttloff ${ }^{1}$, Friedhelm Wawroschek ${ }^{4}$, Holger Gerullis ${ }^{\natural}$

1. Department of Urology, HELIOS Hospital, Bad Saarow, Germany;

2. Department of Urology, Lukaskrankenhaus Neuss, Germany;

3. Uropraxis Dr. Hohmuth, Ulm, Germany;

4. University Hospital for Urology, Klinikum Oldenburg, School of Medicine and Health Sciences, Carl von Ossietzky University Oldenburg, Germany.

$\triangle$ Corresponding author: Dr. Holger Gerullis (MD, PhD). University Hospital for Urology, Klinikum Oldenburg, School of Medicine and Health Sciences, Carl von Ossietzky University Oldenburg, Rahel-Straus-Straße 10, D-26133 Oldenburg, Germany. Telephone: +49-441-4032302, Fax: +49-441-4032303 Email: gerullis.holger@klinikum-oldenburg.de

(c) Ivyspring International Publisher. This is an open access article distributed under the terms of the Creative Commons Attribution (CC BY-NC) license (https://creativecommons.org/licenses/by-nc/4.0/). See http://ivyspring.com/terms for full terms and conditions.

Received: 2016.10.17; Accepted: 2016.12.28; Published: 2017.02.25

\begin{abstract}
Introduction: This study addresses minimally invasive anesthesiologic and analgetic approaches for stone surgery in the upper urinary tract. Aim of this retrospective analysis is to compare feasibility, safety and complication rates of percutaneous nephrolithotomy (PCNL) under local infiltration anesthesia alone (Group I) and additive intravenous analgetics and/or sedative medications (Group II).

Material and Methods: This is a single center study. A total of 439 patients have been included from November 2003 until March 2012. A total of 226 patients were assigned to Group I receiving local infiltration anesthesia alone, whereas 213 patients were assigned to Group II receiving additive intravenous analgetics and/or sedative medications. Demographic characteristics and stone characteristics have been evaluated to determine feasibility, complication rates for safety, and stone-free rates for effectiveness. The study and the reported technique have then been retrospectively analysed according to the IDEAL stages of surgical innovation.

Results: All included patients who accepted local infiltration anesthesia underwent PCNL successfully. The mean American Society of Anesthesiologists score (ASA) of the included patients was $2.15 \pm 0.37$ (range, 1-4). PCNL was indicated in 138 patients due to pelvic calculi, in 171 patients due to renal calculi, in 66 patients due to partial staghorn, in 48 patients due to complete staghorn and in 16 patients due to upper ureteral stones. The total stone free rate in our patients was $78.4 \%$ over all stone localizations. Compared to the possibility of using additive intravenous analgetics and/or sedative medications we could show differences in the median age $(p=0.005)$ suggesting that older patients did better tolerate the infiltration anesthesia than patients at younger ages. We did also remark not statistically significant differences in Group I and Group II as for number of tracts, operation duration, hemoglobin drop, fever, transfusion rate, and stone free rate, but not for severe complications such as perirenal hematoma, colon perforation, pleura perforation, AV fistula, skin fistula, and mortality rate.

Conclusion: PCNL performed under local infiltration anesthesia is a feasible method. It provides satisfactory positive clinical outcomes. Younger age seems to predispose to conversion to extended anesthesiologic procedures. When retrospectively applying the IDEAL criteria, the method can be assigned to the $E$ level or stage $2 b$.
\end{abstract}

Key words: complication, percutaneous nephrolithotomy, local anesthesia, IDEAL. 


\section{Introduction}

Percutaneous nephrolithotomy (PCNL) is an elegant procedure for the treatment of stone disease that has first been described in $1976{ }^{1}$. Nowadays this approach is the first choice of therapy for multiple or single large renal stones ${ }^{2}$. PCNL is also indicated for upper ureteral calculi ${ }^{3}$. Normally, this procedure is performed under general anesthesia or regional anesthesia. Due to improvement in experience and technique, many urologists are interested in performing PCNL under local infiltration anesthesia as recently published ${ }^{4,5}$. After publication of the experience of Li et al. ${ }^{6}$ this is the first European study investigating $\mathrm{PCNL}$ under local infiltration anesthesia.

Up to date the number of PCNL performed at our institution is more than 1800 cases with high focus on procedures in local infiltrating anesthesia.

To make this treatment more comfortable we established a stand-by anesthesia to have the possibility to decide during treatment, if an intravenous application of analgetics and/or sedative medications is necessary or not. Therefore we compared a group of patients without this intravenous application and one group with application.

The aim of this study was to find out differences in the treatment of PCNL in local infiltrating anesthesia with and without stand-by anesthesia. The motivation to perform PCNL in local anesthesia is to have lower complication rates, no complications due to general anesthesia and the possibility to communicate with the patient while operation.

The IDEAL Collaboration (Idea, Development, Exploration, Assessment, Longterm-follow-up) is an open network aiming to enable surgery to develop a solid evidence in order improve outcomes for patients. IDEAL suggests 5 different stages of surgical innovations and encourages adapting the reporting, study planning etc. accordingly. ${ }^{7-9}$. Although in prospective fashion a retrospective analysis of surgical innovations is accepted and can be related to the suggested study design.

\section{Materials and Methods}

\section{Subjects}

In this retrospective study we report on 439 patients (247 men and 192 women, median age 59 years, range 14 to 90) who have been operated between November 2003 and March 2012 at one single institution. Nine patients who received percutaneous nephrolithotomy have been excluded because the treatment had been performed in intubation anesthesia. Our study population consisted of consecutive referrals for therapy of renal stone disease.

\section{Study Design}

All included patients $(n=439)$ underwent PCNL under ropivacaine hydrochloride infiltration anesthesia. Patients were stratified into two groups in this study. Group I $(n=226)$ consists of patients with only local infiltration anesthesia, although intravenous anxiolytics have been allowed. Inclusion criteria for Group II $(n=213)$ were defined as a more active anesthesia with intravenous injection of analgetics and/or sedative medications. Most reasons to include patients into Group II and change to a more active anesthesia were patient disquietness, technical problems, and to anesthesia concerns. In conclusion Group I needed nothing more than local anesthesia and in some cases intravenous anxiolytics; any more medicaments (intravenous analgetics, sedative medications and/or endotracheal anesthesia) were definition criteria for Group II. A flow chart for group assignment is shown in figure 1 .

Inclusion criteria for both groups were: one or more renal stones $>2 \mathrm{~cm}$ in diameter. The exclusion criteria were: intolerance of prone position, irreversible coagulopathy, and intolerance of local infiltration anesthesia. Upper urinary calculi were diagnosed by ultrasound, kidney, ureter, and bladder X-ray, intravenous urography and/or computed tomography (CT) scans. A positive stone sign in X-ray was mandatory. Other imaging like intravenous urography or CT scan have been added in some cases. All patients received urine status, urine culture, and laboratory diagnostics. Full disclosure was given to all participating patients about the operation risks and possibly experiencing short periods of discomfort and pain during surgery.

\section{Surgical procedure of PCNL under local infiltration anesthesia}

Dormicum $(7.5 \mathrm{mg}$ ) premedication was applied one hour before surgery. In the operation theatre patients were positioned in a lateral recumbent position. Patients were monitored for electrocardiography and oxygen saturation during surgery. PCNL was performed under ropivacaine infiltration anesthesia. X-ray was performed to observe the position of the calculi, to decide the puncture site and direction. The puncture site guided by ultrasound was most common below the $12^{\text {th }}$ subcostal space for lower and middle calculi, and above $12^{\text {th }}$ subcostal space for upper calculi, always between the posterior axillary line and scapular line. Ropivacaine $(7.5 \mathrm{mg} / \mathrm{mL})$ was infiltrated with a 22-gauge spinal needle (Chiba needle; Möller Medical, 
Fulda, Germany) from the skin to the renal parenchyma along the puncture direction, including the skin, subcutaneous tissue, muscles, renal capsule, and the underlying parenchyma. The total usage of ropivacaine was $15-20 \mathrm{~mL}$. X-ray-guided percutaneous punctions with an 18-gauge coaxial nephrostomy punction needle (Teleflex Medical, Kamen, Germany) were made into the designed calyx. After the urine efflux was seen, a J-guide wire (coated, $3 \mathrm{~mm}$ J bending, solid core; Peter Pflugbeil, Zorneding, Germany) was inserted into the collecting system. In some cases a second wire (PTFE-coated guidewire; Coloplast, Humlebæk, Denmark) was used due to more security. A $1 \mathrm{~cm}$ skin incision was made, and a suitable size (28 Charrière) working sheath was placed directly as the percutaneous tract (28 Ch working sheath of fascia dilatator, $28 \mathrm{Ch}$ working sheath of dilatator). Under direct nephroscope (26 Ch) (Karl Storz GmbH \& Co. KG, Tuttlingen, Germany) vision, the stone was fragmented by sonotrode system (Karl Storz $\mathrm{GmbH}$ \& Co. KG, Tuttlingen, Germany). The stone fragments were pushed out by a sonotrode or taken out by grasping forceps (Karl Storz GmbH \& Co. KG, Tuttlingen, Germany). After insertion of nephroscope image guidance was made by X-ray with c-arm (Ziehm 8000). The length of the operation was determined according to the size of the stone, the amount of haemorrhage, perfused fluid volume, the hydronephrosis situation, and the vital signs of the patient. Regular endpoint of the operation was stone free status of the patient after endoscopic and X-ray criteria. A plain radiography was performed on the second postoperative day. All X-rays were re-evaluated for this study. For patients with higher BMI a longer nephroscope is available. Finally, a nephrostomy tube $(26 \mathrm{Ch})$ was placed in the pelvis. When patients complained of pain, 5-10 mL of ropivacaine was injected in the capsular puncture site. All operations in this series were performed by a single surgeon (J.R.) who had been fully trained in PCNL. All procedures have been performed in the same center by one surgeon. The surgeon had an experience of 1200 PCNL, when starting this study population. Therefore, it is not possible to show a learning curve.

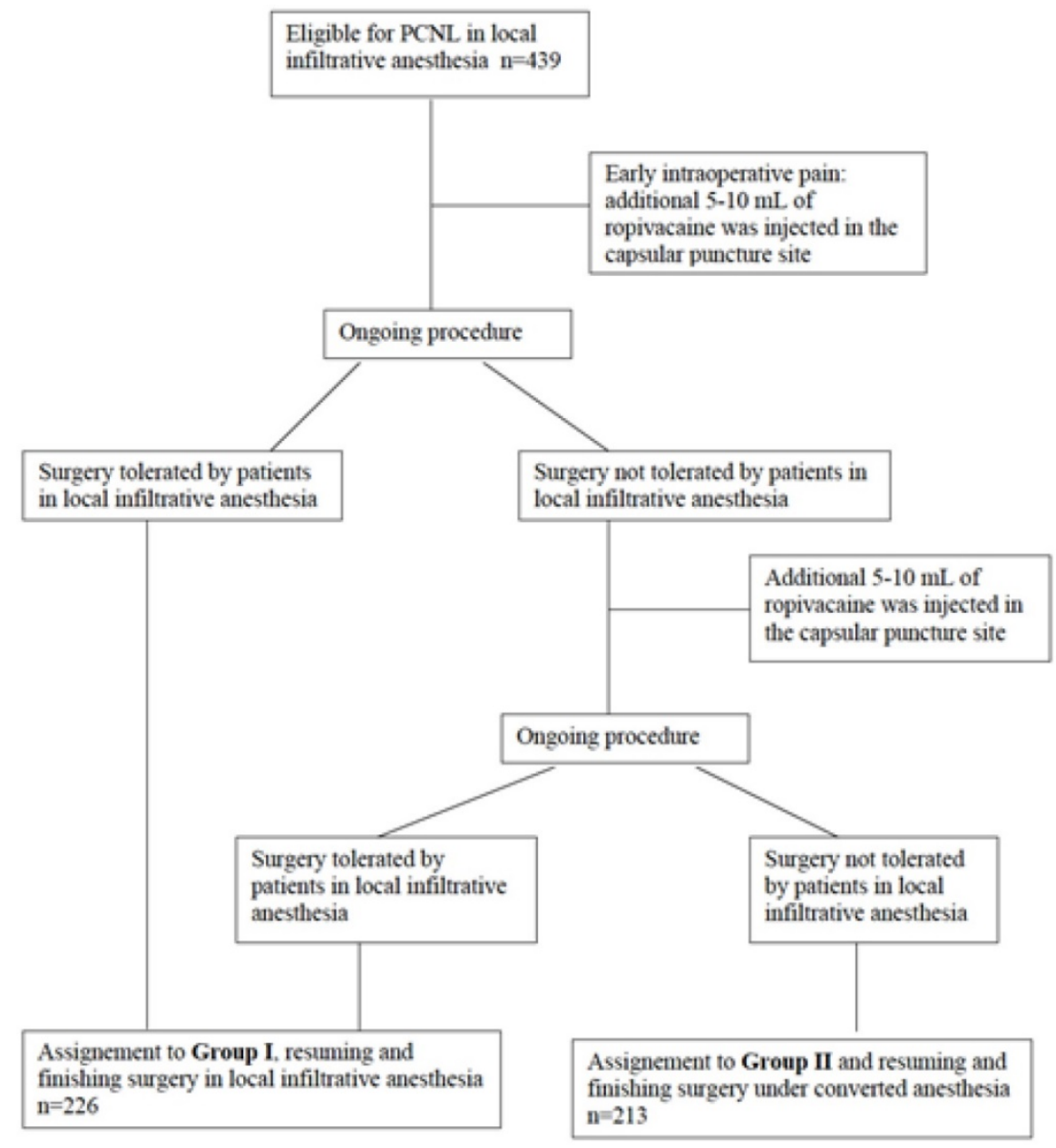

Figure 1: Criteria for group assignment. 


\section{Evaluated Data}

The data evaluated included body mass index (BMI), ASA score, stone position, tract number, duration of surgery, success rate, and surgical complications. All surgical complications have been classified according to the Clavien Dindo classification $^{10}$. The duration of the operation was calculated from the beginning of the local anesthetic infiltration until the completion of placing the nephrostomy catheter. Blood loss was calculated by the hemoglobin level preoperatively and the first postoperative day. A chest X-ray was performed when a recognized intraoperative hydropneumothorax occurred, the physical examination revealed an abnormality or the patient experienced respiratory difficulties in the postoperative period. The success rate was evaluated using plain X-ray and report of surgeon after endoscopy. Success was defined as follows: no remaining stone fragments $>3 \mathrm{~mm}$.

Statistical analysis included Mann-WhitneyU-Test and Chi-square-Test after Pearson's correlation computed and performed by the computer program SPSS version 23 .

\section{Results}

The demographic characteristics are shown in Table I, and main clinical results are shown in Table II. Patients who had not tolerated PCNL in local anesthesia, received general anesthesia. All patients underwent PCNL successfully. All stones were radiopaque. The median ASA score was 2. Pain levels during surgical treatment have been mild and could be tolerated by most patients.

Table I: Demographic characteristics of the patients.

\begin{tabular}{llll}
\hline Parameter & Total & \multicolumn{1}{r}{ Group I } & Group II \\
\hline & No. & & \\
Sex (male / female) & $247 / 192$ & $129 / 97$ & $118 / 95$ \\
Side of stone (left / right) & $271 / 176$ & $132 / 94$ & $132 / 80$ \\
(some patients had bilateral stones) & & & \\
ASA score & & & \\
1 & 28 & 11 & 17 \\
2 & 171 & 88 & 83 \\
3 & 63 & 31 & 32 \\
4 & 3 & 3 & 0 \\
& Median (range) & \\
Age (years) & $59(14-90)$ & $63(27-90)$ & $56(14-84)$ \\
Body mass index $\left(\mathrm{kg} / \mathrm{m}^{2}\right)$ & 27.8 & 27.5 & 28.1 \\
& $(14.7-57.7)$ & $(16.6-57.7)$ & $(14.7-55.5)$ \\
ASA score & $2(1-4)$ & $2(1-4)$ & $2(1-4)$ \\
\hline
\end{tabular}

Comparing Group I and II no statistically significant difference is seen for sex $(p=0.398)$, BMI $(p=0.103)$, ASA $(p=0.259)$, and side of stone $(p=0.234)$, but for age $(p=0.005)$. In the group with only local infiltration anesthesia there are older patients, suggesting that older patients could tolerate this procedure better.

Table II: Main clinical results.

\begin{tabular}{|c|c|c|c|}
\hline Parameter & Total & Group I & Group II \\
\hline & No. (\%) & & \\
\hline \multicolumn{4}{|l|}{ No. of percutaneous tract } \\
\hline 1 & 407 (92.7) & $214(95.1)$ & $192(90.1)$ \\
\hline 2 & $31(7.1)$ & $10(4.5)$ & $21(9.9)$ \\
\hline 3 & $1(0.2)$ & $1(0.4)$ & \\
\hline \multicolumn{4}{|l|}{ No. of transrenal tract } \\
\hline 1 & $369(83.8)$ & $199(87.6)$ & $170(79.8)$ \\
\hline 2 & $50(11.4)$ & $19(8.4)$ & $31(14.6)$ \\
\hline 3 & $9(2.1)$ & $7(3.1)$ & $2(0.9)$ \\
\hline 4 & $6(1.4)$ & $1(0.4)$ & $5(2.3)$ \\
\hline 5 & $4(0.9)$ & & $4(1.9)$ \\
\hline 6 & $1(0.2)$ & & $1(5.5)$ \\
\hline \multicolumn{4}{|l|}{ Fever } \\
\hline None & $384(87.2)$ & $205(90.3)$ & $179(84.0)$ \\
\hline Until 48 hrs after surgery & $35(8.0)$ & $10(4.4)$ & $25(11.7)$ \\
\hline Over 48 hrs after surgery & $20(4.6)$ & $12(5.3)$ & $9(4.2)$ \\
\hline \multicolumn{4}{|l|}{ Other complications } \\
\hline Perirenal hematoma & $2(0.5)$ & $1(0.4)$ & $1(0.5)$ \\
\hline Perforation of colon & $1(0.2)$ & $0(0)$ & $1(0.5)$ \\
\hline Perforation of pleura & $2(0.5)$ & $1(0.4)$ & $1(0.5)$ \\
\hline AV fistula & $2(0.5)$ & $1(0.4)$ & $1(0.5)$ \\
\hline Skin fistula & $1(0.2)$ & 0 & $1(0.5)$ \\
\hline Transfusion & $10(2.3)$ & $5(2.2)$ & $5(2.3)$ \\
\hline Mortality & $0(0)$ & $0(0)$ & $0(0)$ \\
\hline \multicolumn{4}{|c|}{ Complication after CLAVIEN classification } \\
\hline 0 & $363(82.7)$ & $195(86.3)$ & $168(78.9)$ \\
\hline 1 & $58(13.2)$ & $21(9.3)$ & $37(17.4)$ \\
\hline 2 & $12(2.7)$ & $7(3.1)$ & $5(2.3)$ \\
\hline 3 & $6(1.4)$ & $3(1.3)$ & $3(1.4)$ \\
\hline 4 & $0(0)$ & $0(0)$ & $0(0)$ \\
\hline \multirow[t]{2}{*}{5} & $0(0)$ & $0(0)$ & $0(0)$ \\
\hline & Median (range) & & \\
\hline Duration of operation (min) & $\begin{array}{l}42 \\
(9-250)\end{array}$ & $\begin{array}{l}39.5 \\
(13-137)\end{array}$ & $\begin{array}{l}48 \\
(9-250)\end{array}$ \\
\hline Hemoglobin drop (mmol/L) & $\begin{array}{l}0.7 \\
(-0.6-4.9)\end{array}$ & $\begin{array}{l}0.7 \\
(-0.6-4.9)\end{array}$ & $\begin{array}{l}0.8 \\
(-0.5-3.5)\end{array}$ \\
\hline
\end{tabular}

The main clinical results are shown in table II. The number of percutaneous tracts reaches from 1 to 3 . There is a trend of more percutaneous tracts when PCNL was performed with intravenous sedative medications, but no statistical significance $(p=0.056)$. For transrenal tracts (range, 1-6) there is a statistical significance calculated with $p=0.010$ with more tracts in Group II. Percutaneous tracts are performed by puncture of skin and kidney, while transrenal tract means only different punctures of the kidney via a single skin whole. The median operative time was 42 minutes (range, 9-250). There was a statistically significant difference with $p<0.005$ in the median duration of the operation in Group I (39.5 minutes) in comparison to Group II (48 minutes). This difference could be explained by the additional time consumed by the conversion of the anesthesiologic procedure.

The median hemoglobin drop was $0.7 \mathrm{mmol} / \mathrm{L}$ (range, -0.6-4.9). The mean hemoglobin drop in Group 
II was higher with $0.8 \mathrm{mmol} / \mathrm{L}$ compared with Group I, and also statistically significant with a calculated $p$-value of $p=0.011$. Regarding the rates of fever after treatment there is a higher rate of fever in Group II with a statistically significance of $p=0.031$. In total ten patients $(2.3 \%)$ received transfusion, nearly same rates in both groups $(p=0.613)$. Also, all other severe complications have a similar frequency in both groups, and therefore there is no statistically difference for perirenal hematoma $(p=0.736)$, colon perforation $(p=0.485)$, pleura perforation $(p=0.736)$, AV fistula $(p=0.736)$, and skin fistula $(p=0.485)$. Mortality is zero in both groups.

To have a higher transparence for surgical complications the Clavien Dindo classification was used. There is a trend for higher complication rates in Group II, but there is no statistical significance $(p=0.095)$. In total we had six complications grade 3 after Clavien Dindo classification, three complications in Group I (one case of arterio-venous fistulation, two cases of vesical tamponade), three complications in Group II (vesical tamponade, pneumothorax, bowel injury).

One of the main reasons why intravenous medications were needed was the incommodious position to lie while treatment was performed.

The total stone free rate in patients was $78.4 \%$ over all stone localizations. Details of stone free rates for different localizations in both groups are shown in Table III. Surprisingly, the stone free rate is statistically significant higher in Group I $(p<0.005)$.

This study represents a comparative analysis of two groups for a technique which had been reported earlier from different surgeons in comparative or even higher quality ${ }^{6}$.

It corresponds to numerous of the requirements of the Exploration Stage, $2 b$ according to IDEAL although not being prospective in nature, However, at the current stage of IDEAL integration in current surgical research reporting methods, discussing and classifying even retrospective studies helps to spread the IDEAL recommendations and attributes future comparability patterns for reported techniques.

\section{Discussion}

The indication of PCNL under local infiltration anesthesia is similar to PCNL under regional and general anesthesia ${ }^{11,12}$. ASA score of our cohort is in the same range than PCNL in regional or general anesthesia. In fact, cases with contraindications for epidural and/or general anesthesia are often performed in local infiltrative anesthesia. Especially patients with higher ASA score 3 and 4 are high risk patients for regional and/or general anesthesia; those patients are also included in this study and have been operated successfully. In our study we retrospectively compared local infiltrative anesthesia with ropivacaine alone and additive intravenous injection of analgetics and/or sedative medications. It has been shown before that ropivacaine is more effective for infiltration of the nephrostomy tract than bupivacaine ${ }^{13}$.

The presence of pain during PCNL may be a limitation factor for the use of local infiltrating anesthesia for PCNL. In 21 cases this procedure could not be performed due to different circumstances, e.g. not tolerating pillow, fever, anxiety etc. The pain during PCNL is caused mainly by the dilatation of the renal capsule and parenchyma but not stone disintegration ${ }^{4}$. The key to prevent severe pain during surgery is the appropriate dosage of local anesthetic administered along the entire tract from the skin to the capsular puncture site and the underlying parenchyma. Preoperative explanation is also important to bring good perioperative cooperation which may help to raise the pain threshold of patients during surgery.

The major complications presented by patients were fever, bleeding, and renal collecting system injury. The incidences of fever reported in the literature vary from 1 to $32.1 \% 3,14,15$. Urine analysis and culture were performed preoperatively for all patients; the study also required mandatorily prophylactic antibiotics and draining of a pyonephrotic kidney before performing PCNL ${ }^{3}$. The fever rate in the study was low; maybe this is caused due to the observation of the shivering of patients.

Table III: Localisation of stones and stone free rates in bold numbers.

\begin{tabular}{|c|c|c|c|c|c|c|}
\hline \multirow[t]{2}{*}{ Localization } & \multicolumn{2}{|l|}{ Total } & \multicolumn{2}{|l|}{ Group I } & \multicolumn{2}{|l|}{ Group II } \\
\hline & No. (\%) & Stone-free rate no. $(\%)$ & No. (\%) & Stone-free rate no. $(\%)$ & No (\%) & Stone-free rate no. $(\%)$ \\
\hline Pelvic calculi & $137(31.3)$ & $125(90.6)$ & $76(34.1)$ & $72(93.5)$ & $61(28.4)$ & $53(86.9)$ \\
\hline Renal calculi & $172(39.2)$ & $147(85.0)$ & $105(46.9)$ & $94(88.7)$ & $67(31.2)$ & $53(79.1)$ \\
\hline Partial staghorn & $66(15)$ & $45(68.2)$ & $25(5.7)$ & $18(72)$ & $41(19.1)$ & $27(65.9)$ \\
\hline Complete staghorn & $48(10.9)$ & $11(22.9)$ & $11(4.9)$ & $4(36.4)$ & $37(17.2)$ & $7(18.9)$ \\
\hline Upper ureteral & $16(3.7)$ & $16(\mathbf{1 0 0 )}$ & $7(3.1)$ & 7 (100) & $9(4.2)$ & $9(100)$ \\
\hline
\end{tabular}


Bleeding can be an important complication in PCNL. Compared with $1-12 \%$ of patients requiring a blood transfusion 16 , and $0.15-1.4 \%$ of patients receiving renal vessel embolization to control hemorrhage $3,17,18$ in previous reports, $2.3 \%$ of patients requiring transfusion and $0.5 \%$ of patients receiving selective renal vessel embolization were acceptable in the study.

Pleural injury was another important complication for PCNL especially for patients with supra $12^{\text {th }}$ rib access. Of the patients who accepted postoperative chest X-ray, there were only two patients with hydrothorax that required drainage. Some studies reported colonic injury as well as pleural injury ${ }^{14}$.

Although single tract PCNL is an efficient procedure to clear renal stones ${ }^{19}$, the study also applied multiple tracts to handle complex stones. PCNL monotherapy using multiple tracts is safe and effective and should be the first option for massive renal staghorn calculi ${ }^{20}$. The study established a maximum of 6 tracts for the patients with complex stones; the results demonstrated that multiple tracts under local anesthesia are feasible; however in this study 3 or more tracts were used for only $4.6 \%$ of the patients. Therefore, the use of more than 2 tracts under local anesthesia was only considered for the patients who had complex kidney stones, a high pain threshold, and who could have the tracts placed quickly. The higher numbers of tracts are more common in cases with additive intravenous anesthesia.

Current literature reports stone-free rates after PCNL from $40-90 \%$, depending on the size, number, composition, and nature of the stone and the surgeon's experience ${ }^{21}$. The total stone-free rate of this series was $78.4 \%$, similar to the result in the research literature. The term of clinically insignificant residual fragments is controversial, but is generally accepted as residual fragments smaller than 2 to $5 \mathrm{~mm}$ 22,23 . As stone free rate is considered overestimated with radiography, we didn't use CT scan for stone free rate evaluation ${ }^{24}$. The study could show that in Group I the stone free rate is significantly higher. This may be caused by a higher rate of complicated stones with more tracts in Group II.

Although no serious complication occurred in this series, one of the shortcomings of local infiltration anesthesia is that immediate change into open surgery is difficult. Therefore, the surgeon must be fully trained with PCNL and able to prevent the serious complications. According to the published data, competence at performing PCNL is reached after 60 cases and excellence after 115 cases $^{25,26}$. Therefore, it is recommended that a surgeon performs PCNL under local infiltration anesthesia after successfully completing 120 cases of PCNL under epidural anesthesia or general anesthesia. Patient fear and anxiety during the surgical procedure is an additional problem that requires consideration. It is necessary that the patients are given full disclosure about the local infiltration anesthesia and the surgery. If local infiltration anesthesia is not tolerated by the patient, other ways of anesthesia should be considered.

In this study the median BMI is high with 27.8 $\mathrm{kg} / \mathrm{m}^{2}$, the rate of obese patients (BMI $>30.0 \mathrm{~kg} / \mathrm{m}^{2}$ ) was high with $32.8 \%$. In the Chinese study of Li et al., the BMI was lower with a mean BMI of $24.8 \mathrm{~kg} / \mathrm{m}^{2}$, and only $5.5 \%$ of the patients were obese ${ }^{6}$. However, BMI seems not to negatively influence safety and feasibility of the procedure.

We could compare the cohort of patients with real local infiltrative anesthesia with or without anxiolysis, and the cohort of patients who needed intravenous pain management and/or analgosedation, but no intubation. Another limitation is that only 1 pain-assessment and monitoring tool was used to assess tolerability of the procedure. Therefore, comparative studies should be performed to validate the study results, and more pain-assessment and monitoring tools should be used to measure intraoperative and postoperative pain.

There is a trend for higher complication rates after Clavien Dindo in Group II, but there is no statistical significance $(p=0.095)$. Probably one of the reasons why the complication rate is lower in the group with only local infiltrating anesthesia could be caused by compulsion that treatment is only possible in the previous infiltrated area. Main advantages of this technique are accessibility of patients with elevated ASA score and the possibility to communicate with the patient during the entire surgery.

The introduction of new surgical methods, surgical innovations or variations as well as medical devices do not yet follow clear paradigms like is the case in the introduction process of new drugs. Therefore, a new approach, the IDEAL method has been proposed in 2009 by Mc Culloch and colleagues 8. IDEAL clearly provides stages of surgical innovations which allow assigning every method to its particular level of development and evidence. In 2016 the IDEAL criteria have been extended to IDEAL-D for medical devices in order to improve product surveillance, even at preclinical stages of development in order to rationalize regulatory structures and to minimize regulatory delay 27.

As the IDEAL approach is new and not commonly introduced into the surgical science yet, to date the amount of prospective data collected in order 
to classify this particular surgical method according to IDEAL is limited. However, retrospective data as in the current study may contribute to the evaluation of the method according to IDEAL. Our particular study adds a group of 226 patients with PCNL under local infiltration anesthesia to the already existing number of 2000 patients reported by Li et al. ${ }^{6}$. Thus, according to IDEAL, PCNL in local infiltration anesthesia may be assigned to the Exploration stage (E). The preferred setting to achieve the $\mathrm{E}$ status according to IDEAL would be a prospective collaborative observational study or a feasibility randomized controlled trial. However, the current study as well as the comparable study by $\mathrm{Li}$ et al. ${ }^{6}$ are retrospective in nature which does not interdict to assign them to the IDEAL model as the steps are clearly defined. In addition, the IDEAL criteria have been first published in 2009 and increasingly used ever since. The method presented in this report has been performed in patients beginning in 2003, thus 6 years before official announcement of the IDEAL criteria.

With regard to future development of this technique it seems unlikely to enroll a prospective randomized trial in order to compare outcome and effectiveness of this method to current standard methods. The IDEAL recommendations are aware of this difficult situation of randomized trials in surgical procedures and provide with alternative designs to fulfill stage 3 requirements as observational studies or interrupted time series which would represent an appropriate approach to further evaluate this method. To fulfil stage 4 according to IDEAL a registry for this method should be implemented. This would enable surgeons using this method to register their data and experiences when applying this method.

However, there are more different instruments to objectively monitor and control quality of surgical practices and techniques which is necessary in order to avoid periods of suboptimal performances, in particular if an agreed standard regarding a particular technique already exists. The cumulative sum (CUSUM) is a useful tool for surveillance and quality control. In particular for studies of surgical procedures that aim at transparency according to IDEAL the cumulative sum could be useful when analyzing operative times, necessary conversions, intraoperative complications and respective adaptions. The application of CUSUM or in particular for surgical procedures: the learning curve-cumulative summation (LC-CUSUM) would have been helpful in this study to analyse e.g. time to proficiency and maintenance of competence in this particular technique ${ }^{28}$. In addition, the respective surgeon had previously, during years of clinical practice and experience trained and performed this technical procedure of stone surgery more than 1200 times, thus the novelty has been the analgesetic approach. However, the study was not prospective in nature thus LC-CUSUM has not been applied.

Assigning this method to the E stage states its current status of evidence and suggests algorithms for future trial designs in order to investigate the method and prove its position in current stone surgery.

\section{Conclusion}

Local infiltration anesthesia is a well-tolerated alternative anesthesia technique that provides effective intraoperative analgesia for PCNL.

PCNL performed under local infiltration anesthesia is feasible and provides satisfactory positive clinical outcomes. This study shows that both kinds of treatment are similar in success and complication rates. Younger age seems to predispose towards conversion to invasive anesthesia. The method can be assigned to the E level according to IDEAL. Additional comparative studies should be performed to classify efficacy, safety, tract quantity, dilatation method, and the best candidates for this procedure.

\section{Competing Interests}

The authors have declared that no competing interest exists.

\section{References}

1. Fernstrom I, Johansson B. Percutaneous pyelolithotomy. A new extraction technique. Scand J Urol Nephrol 1976;10:257-9.

2. Pugh JW, Canales BK. New instrumentation in percutaneous nephrolithotomy. Indian J Urol 2010;26:389-94.

3. Mousavi-Bahar SH, Mehrabi S, Moslemi MK. Percutaneous nephrolithotomy complications in 671 consecutive patients: a single-center experience. Urol J 2011;8:271-6.

4. Dalela D, Goel A, Singh P, Shankhwar SN. Renal capsular block: a novel method for performing percutaneous nephrolithotomy under local anesthesia. J Endourol 2004;18:544-6.

5. Aravantinos E, Karatzas A, Gravas S, Tzortzis V, Melekos M. Feasibility of percutaneous nephrolithotomy under assisted local anaesthesia: a prospective study on selected patients with upper urinary tract obstruction. Eur Urol 2007;51:224-7; discussion 8 .

6. Li H, Xu K, Li B, Chen B, Xu A, Chen Y, et al. Percutaneous nephrolithotomy under local infiltration anesthesia: a single-center experience of 2000 Chinese cases. Urology 2013;82:1020-5.

7. Barkun JS, Aronson JK, Feldman LS, Maddern GJ, Strasberg SM, Altman DG, et al. Evaluation and stages of surgical innovations. Lancet 2009;374:1089-96.

8. McCulloch P, Altman DG, Campbell WB, Flum DR, Glasziou P, Marshall JC, et al. No surgical innovation without evaluation: the IDEAL recommendations. Lancet 2009;374:1105-12.

9. Ergina PL, Cook JA, Blazeby JM, Boutron I, Clavien PA, Reeves BC, et al. Challenges in evaluating surgical innovation. Lancet 2009;374:1097-104.

10. Dindo D, Demartines N, Clavien PA. Classification of surgical complications: a new proposal with evaluation in a cohort of 6336 patients and results of a survey. Ann Surg 2004;240:205-13.

11. Preminger GM, Tiselius HG, Assimos DG, Alken P, Buck AC, Gallucci M, et al. 2007 Guideline for the management of ureteral calculi. Eur Urol 2007;52:1610-31.

12. Preminger GM, Assimos DG, Lingeman JE, Nakada SY, Pearle MS, Wolf JS, Jr. Chapter 1: AUA guideline on management of staghorn calculi: diagnosis and treatment recommendations. J Urol 2005;173:1991-2000.

13. Parikh GP, Shah VR, Vora KS, Parikh BK, Modi MP, Kumari P. Ultrasound guided peritubal infiltration of $0.25 \%$ Bupivacaine versus $0.25 \%$ Ropivacaine for postoperative pain relief after percutaneous nephrolithotomy: A prospective double blind randomized study. Indian J Anaesth 2014;58:293-7. 
14. Michel MS, Trojan L, Rassweiler JJ. Complications in percutaneous nephrolithotomy. Eur Urol 2007;51:899-906; discussion

15. Bryniarski P, Paradysz A, Zyczkowski M, Kupilas A, Nowakowski K, Bogacki R. A randomized controlled study to analyze the safety and efficacy of percutaneous nephrolithotripsy and retrograde intrarenal surgery in the management of renal stones more than $2 \mathrm{~cm}$ in diameter. J Endourol 2012;26:52-7.

16. Seitz C, Desai M, Hacker A, Hakenberg OW, Liatsikos E, Nagele U, et al. Incidence, prevention, and management of complications following percutaneous nephrolitholapaxy. Eur Urol 2012;61:146-58.

17. Richstone L, Reggio E, Ost MC, Seideman C, Fossett LK, Okeke Z, et al. First Prize (tie): Hemorrhage following percutaneous renal surgery: characterization of angiographic findings. J Endourol 2008;22:1129-35.

18. Srivastava A, Singh KJ, Suri A, Dubey D, Kumar A, Kapoor R, et al. Vascular complications after percutaneous nephrolithotomy: are there any predictive factors? Urology 2005;66:38-40.

19. Shahrour K, Tomaszewski J, Ortiz T, Scott E, Sternberg KM, Jackman SV, et al. Predictors of immediate postoperative outcome of single-tract percutaneous nephrolithotomy. Urology 2012;80:19-25

20. Aron M, Yadav R, Goel R, Kolla SB, Gautam G, Hemal AK, et al. Multi-tract percutaneous nephrolithotomy for large complete staghorn calculi. Urol Int 2005;75:327-32

21. Park J, Hong B, Park T, Park HK. Effectiveness of noncontrast computed tomography in evaluation of residual stones after percutaneous nephrolithotomy. J Endourol 2007;21:684-7.

22. Altunrende F, Tefekli A, Stein RJ, Autorino R, Yuruk E, Laydner H, et al. Clinically insignificant residual fragments after percutaneous nephrolithotomy: medium-term follow-up. J Endourol 2011;25:941-5.

23. Acar C, Cal C. Impact of Residual Fragments following Endourological Treatments in Renal Stones. Adv Urol 2012;2012:813523.

24. Kim JW, Chae JY, Oh MM, Park HS, Moon du G, Yoon CY. Computed tomography-based novel prediction model for the stone-free rate of ureteroscopic lithotripsy. Urolithiasis 2014;42:75-9.

25. Tanriverdi O, Boylu U, Kendirci M, Kadihasanoglu M, Horasanli K, Miroglu C. The learning curve in the training of percutaneous nephrolithotomy. Eur Urol 2007;52:206-11

26. Allen D, O'Brien T, Tiptaft R, Glass J. Defining the learning curve for percutaneous nephrolithotomy. J Endourol 2005;19:279-82.

27. Sedrakyan A, Campbell B, Merino JG, Kuntz R, Hirst A, McCulloch P. IDEAL-D: a rational framework for evaluating and regulating the use of medical devices. BMJ 2016 Jun 6;353:i2372.

28. Biau DJ, Williams SM, Schlup MM, Nizard RS, Porcher R. Quantitative and individualized assessment of the learning curve using LC-CUSUM. Br J Surg 2008;95:925-9. 